\title{
Chaotic dynamics of a Bose-Einstein condensate coupled to a qubit
}

\author{
J. Martin, ${ }^{1,2,3}$ B. Georgeot, ${ }^{1,2}$ and D. L. Shepelyansky ${ }^{1,2}$ \\ ${ }^{1}$ Université de Toulouse; UPS; Laboratoire de Physique Théorique (IRSAMC); F-31062 Toulouse, France \\ ${ }^{2}$ CNRS; LPT (IRSAMC); F-31062 Toulouse, France \\ ${ }^{3}$ Institut de Physique Nucléaire, Atomique et de Spectroscopie, Université de Liège, 4000 Liège, Belgium \\ (Received 30 December 2008; revised manuscript received 31 March 2009; published 12 June 2009)
}

\begin{abstract}
We study numerically the coupling between a qubit and a Bose-Einstein condensate (BEC) moving in a kicked optical lattice using Gross-Pitaevskii equation. In the regime where the BEC size is smaller than the lattice period, the chaotic dynamics of the BEC is effectively controlled by the qubit state. The feedback effects of the nonlinear chaotic BEC dynamics preserve the coherence and purity of the qubit in the regime of strong BEC nonlinearity. This gives an example of an exponentially sensitive control over a macroscopic state by internal qubit states. At weak nonlinearity quantum chaos leads to rapid dynamical decoherence of the qubit. The realization of such coupled systems is within reach of current experimental techniques.
\end{abstract}

DOI: 10.1103/PhysRevE.79.066205

PACS number(s): 05.45.Mt, 37.10.Jk, 42.50.Dv, 67.85.Hj

The dynamics of Bose-Einstein condensates (BECs) in the regime of quantum chaos is now actively investigated by several experimental groups in the world [1-4]. These experiments implement the quantum version of the Chirikov standard map (kicked rotator) [5] by means of kicked optical lattices. This model exhibits interesting phenomena such as dynamical localization of diffusive energy growth, which has been first observed in cold atoms [6]. However, for cold atoms the dynamics is described by the linear Schrödinger equation, while for BEC nonlinear effects are present that can be studied in a wide range of situations with GrossPitaevskii equation (GPE) [7]. Due to this nonlinearity the dynamics of BEC can become truly chaotic with appearance of exponential instability [8,9], provided the size of BEC is smaller than the optical lattice period. In many cases, a correct description of the system is obtained by accounting for the internal structure of the atoms forming the BEC. Such BEC must be seen as quantum objects with several components. In the situation where there are two components, the system becomes equivalent to a BEC coupled to a qubit. This leads to the fundamental problem of the dynamics of a truly chaotic BEC coupled to a qubit and controlled by it. At the same time, this dynamics generates a feedback on the purity and evolution of the qubit, which may significantly affect its properties. Since the BEC dynamics is exponentially unstable, it has the potential to become a detector with exponential sensitivity to the qubit state. The spinor states of BEC are now actively studied experimentally (see, e.g., [10,11]), such that in principle it would allow one to realize the coupling between a qubit and chaotic BEC dynamics. The decoherence of a qubit coupled to a BEC has also been discussed in the context of solid-state physics devices [12].

To describe the dynamics of a BEC coupled to a qubit in a kicked optical lattice we use GPE [7],

$$
\begin{aligned}
i \hbar \frac{\partial}{\partial t}\left(\begin{array}{c}
\psi_{1} \\
\psi_{0}
\end{array}\right)= & \left(-\frac{\hbar^{2}}{2 m} \frac{\partial^{2}}{\partial x^{2}}+\left(k+\varepsilon \sigma_{z}\right) \cos (\kappa x) \delta_{T}(t)+\delta \sigma_{x}\right)\left(\begin{array}{l}
\psi_{1} \\
\psi_{0}
\end{array}\right) \\
& -\left(\begin{array}{c}
\left(g_{11}\left|\psi_{1}\right|^{2}+g_{10}\left|\psi_{0}\right|^{2}\right) \psi_{1} \\
\left(g_{01}\left|\psi_{1}\right|^{2}+g_{00}\left|\psi_{0}\right|^{2}\right) \psi_{0}
\end{array}\right) .
\end{aligned}
$$

The first term on the right-hand side (rhs) accounts for the free evolution of the cold atoms, the second one represents the effect of the optical lattice of period $\lambda=2 \pi / \kappa, \delta_{T}(t)$ being a periodic delta-function with period $T$, and the third one corresponds to the driving of the qubit with a strength $\delta$. The last term describes the nonlinear interactions between atoms treated in the framework of GPE for spinor BEC. We start considerations with the case $g_{11}=g_{10}=g_{01}=g_{00}=g$, where the nonlinear parameter $g=N g_{1 \mathrm{D}}$ is determined by the number $N$ of atoms in the condensate and the effective one-dimensional coupling constant $g_{1 \mathrm{D}}=-2 a_{0} \hbar \omega_{\perp}$, with $\omega_{\perp}$ as the radial trap frequency and $a_{0}$ as the three-dimensional scattering length. The spinor wave function is normalized to one $\left(\int_{\mathbb{R}}\left[\left|\psi_{0}(x)\right|^{2}+\left|\psi_{1}(x)\right|^{2}\right] d x=1\right)$. In the following, we express the momentum of atoms in recoil units and time $t$ in units of the kick period $T$ by setting $\hbar=m=\kappa=1$ in Eq. (1). In the absence of atom-atom interactions at $g=0$, Eq. (1) reduces to the usual kicked rotator model with classical chaos parameter $K=k T$ and effective Planck constant $T$ (see, e.g., $[5,9]$ ). The first three terms on the rhs correspond to the linear evolution of the kicked rotator coupled to a qubit. The strength of the kick depends on the orientation of the qubit due, for example, to different detunings between the atomic transition frequencies and the laser frequency [6]. The qubit physically appears as description of internal atomic states, e.g., two hyperfine states. This linear model was studied in $[13,14]$. In particular, in [14] it was shown that quantum chaos in the kicked rotator leads to rapid decoherence of the qubit. Here we study the effects of a nonlinearity $g$ induced by interactions between atoms in the BEC with chaotic dynamics.

We consider an initial state of the form $|\Psi\rangle=|\psi\rangle \otimes|\phi\rangle$, where $|\phi\rangle=\alpha|0\rangle+\beta|1\rangle$ is the internal state of the BEC and $|\psi\rangle$ its motional state given by the soliton distribution,

$$
\psi(x, t)=\frac{\sqrt{g_{0}}}{2} \frac{\exp \left[i p_{0}\left(x-x_{0}-p_{0} t / 2\right)+i g_{0}^{2} t / 8\right]}{\cosh \left[g_{0}\left(x-x_{0}-p_{0} t\right) / 2\right]},
$$

taken at time $t=0$. For $k=0$ and $g_{0}=g$ this is the exact solution of Eq. (1), which describes the propagation of a soliton with constant velocity $p_{0}[15]$. Numerical simulations are performed using the second-order SABA integrator [16] for 


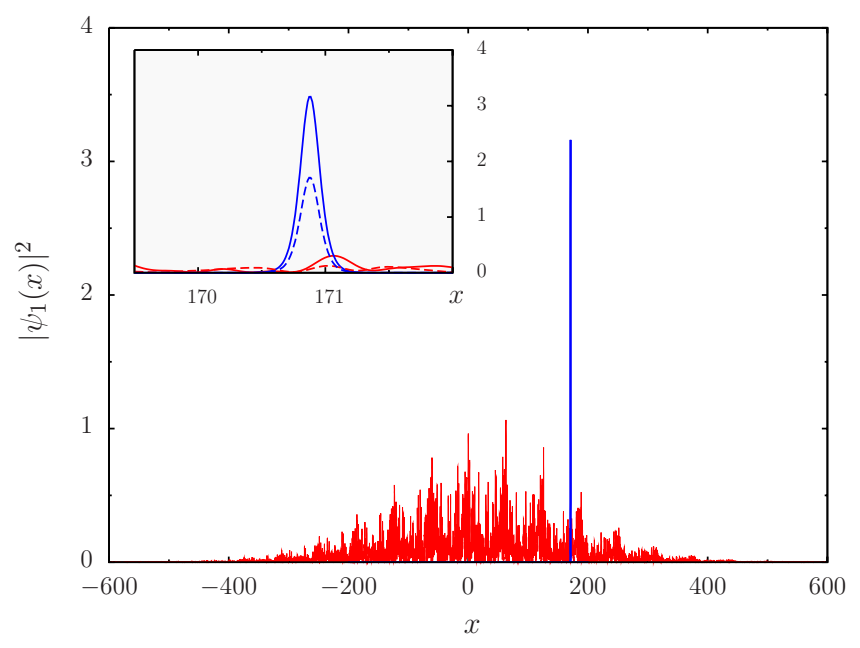

FIG. 1. (Color online) Probability density $\left|\psi_{1}(x)\right|^{2}$ at $t=10$ for $k=3.4, \varepsilon=0.5, T=2$, and $\delta=0.2$ showing a broad distribution at $g=0$ (red/gray curve, magnified by a factor 100) and a BEC soliton at $g=20$ (blue/black curve). The initial state is given by Eq. (2) at $t=0$ for $g_{0}=20, x_{0}=0.1$, and $p_{0}=0.05$ and with qubit state $|\phi\rangle=(|0\rangle+|1\rangle) / \sqrt{2}$. Inset shows data on a smaller scale near the soliton center, with full curves for $\left|\psi_{1}(x)\right|^{2}$ and dashed curves for $\left|\psi_{0}(x)\right|^{2}$.

which the error scales as $\mathcal{O}\left(\Delta t^{3}\right)$ with time step $\Delta t$. Computations have been done with $\Delta p=1 / 64$, total number of states $N_{S}=2^{15}-2^{16}$, and time step $\Delta t \approx 0.01 /(g+1)$.

At moderate values of $k$, a narrow distribution with solitonic form (2) spreads rapidly in space and momentum in absence of interactions between atoms $(g=0)$. In contrast, for sufficiently large $g$, the shape of the soliton is only slightly perturbed even after a large number of kicks (see Fig. 1). In this regime, the center $\left(x_{c}, p_{c}\right)$ of the BEC follows the dynamics described by Chirikov standard map [8]: $\bar{p}_{c}=p_{c}$ $+k \sin x_{c}, \bar{x}_{c}=x_{c}+\bar{p}_{c} T$, where bars denote the values of the soliton position and velocity after one kick iteration. Accordingly, the soliton dynamics becomes chaotic for $K=k T \gtrsim 1$. In presence of the qubit dynamics imposed by the Rabi splitting $\delta$, the kick function $f\left(x_{t}\right)=\left(x_{t+1}-2 x_{t}+x_{t-1}\right) /(k T)$ starts to depend on the internal qubit state (here $x_{t}$ is the average position of all atoms from the two components at time $t$ ). This leads to a splitting of $f$ amplitude between the two values $(1 \pm \varepsilon / k) \sin x$ determined by the qubit state. In presence of Rabi splitting $\delta$, the amplitude $f$ varies between these two limiting values due to nontrivial qubit dynamics as is clearly seen in Fig. 2. Due to the qubit oscillations, the soliton can even penetrate inside classically integrable regions (see inset of Fig. 2). Inside the chaotic region a soliton trajectory in the phase space is exponentially sensitive to the qubit internal quantum state.

The GPE nonlinearity $g$ also influences strongly the qubit dynamics. Indeed, for $g=0$ the purity of the qubit $\xi(t)$ $=|\operatorname{Tr}[\rho(t) \vec{\sigma}]|$ decays rapidly to almost zero due to quantum chaos in the dynamics of atoms, as can be seen in Fig. 3. The decay is linked to the Lyapunov exponent of classical chaotic dynamics as discussed in [14]. In contrast, for large values of $g$, the purity of the qubit decays very slowly, remaining close to 1 after almost 100 kicks (see Fig. 3). In this regime, the

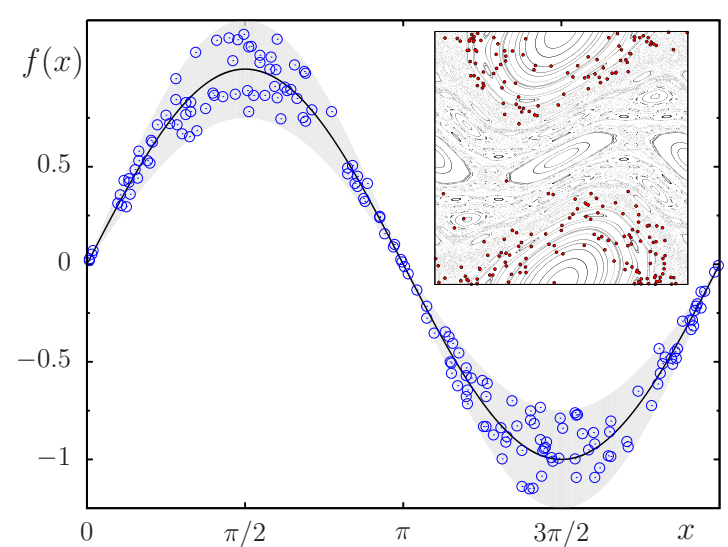

FIG. 2. (Color online) Circles show the kick function $f(x)$ obtained from the BEC dynamics of Eq. (1) with parameters $k=1$, $\varepsilon=0.25, T=4, \delta=0.2$, and $g=20$. Initial state is the same as in Fig. 1. The black solid line shows $f(x)=\sin x$, while the gray area, induced by the qubit, corresponds to the region delimited by the curves $(1 \pm \varepsilon / k) \sin x$. Inset: Poincaré section of the standard map at $K=1$ (small dots); BEC positions are shown by large red/gray dots for parameters $k=1, \varepsilon=0.5, T=1, \delta=0.05$, and $g=20$ and initial state as in the main figure.

purity decay is determined by the decay of the solitonic part of the BEC. Indeed, the latter can be defined as $W_{s}=\int_{x_{c}-10 / g}^{x_{c}+10 / g}\left[\left|\psi_{0}(x)\right|^{2}+\left|\psi_{1}(x)\right|^{2}\right] d x$ with $x_{c}$ taken as the soliton center position, and the data in Fig. 3 show that it decays in the same way as the purity $\xi$. Since the lifetime of the soliton

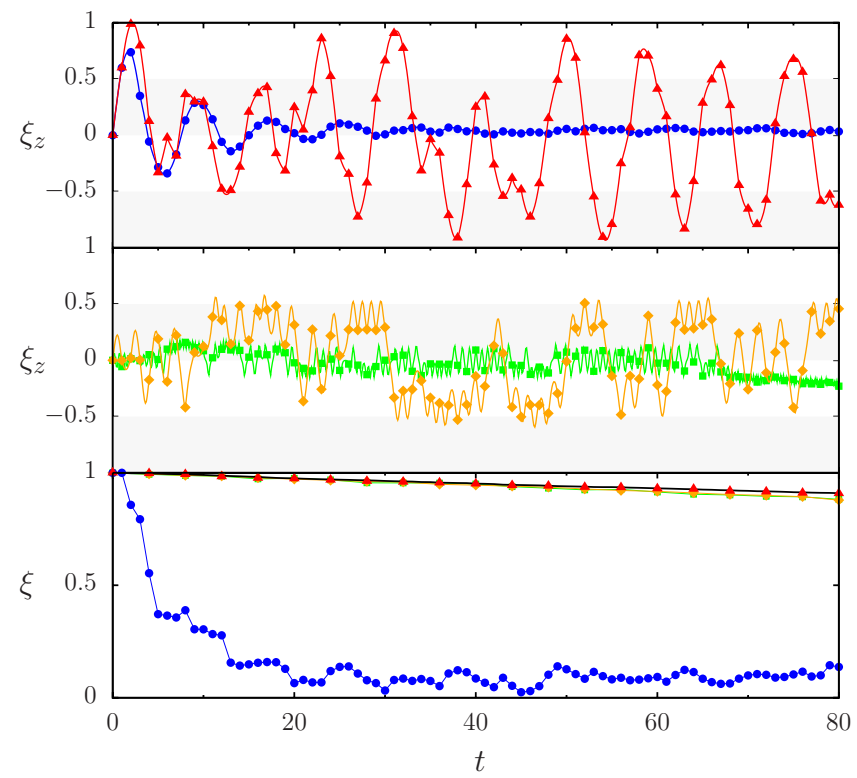

FIG. 3. (Color online) Qubit polarization $\xi_{z}$ (top and middle panels) and purity $\xi$ (bottom panel) as a function of time for $k=3.4, \varepsilon=0.5, T=2$, and $\delta=0.2$. Data are shown for (top panel) $g_{11}=g_{10}=g_{01}=g_{00}=g$ with $g=20$ (red triangles) and $g=0$ (blue circles) and (middle panel) $g_{00}=g_{11}=21$ and $g_{01}=g_{10}=19$ (orange diamonds) and $g_{00}=g_{11}=30$ and $g_{01}=g_{10}=10$ (green squares). In the bottom panel, the black solid line shows the solitonic part $W_{s}$ (see text); other symbols are as in top and middle panels. Initial state is the same as in Fig. 1. 


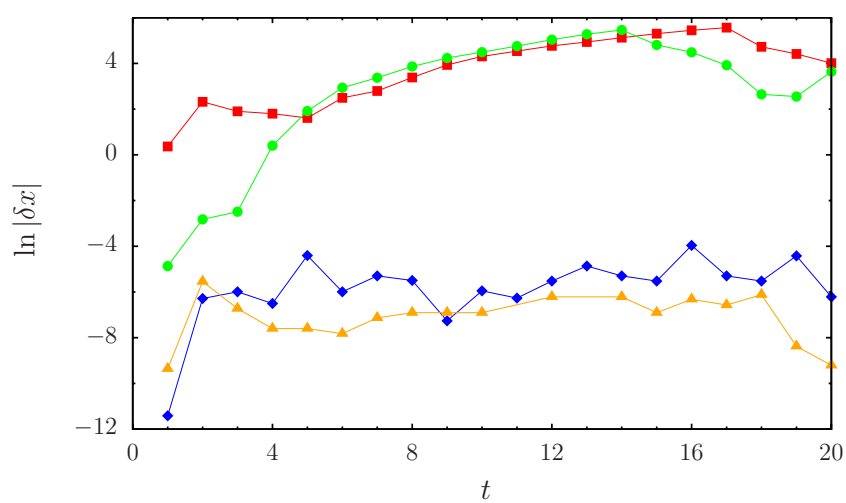

FIG. 4. (Color online) Distance $\delta x$ between solitons with the qubit in the states up $\psi_{1}(x, t)$ and down $\psi_{0}(x, t)$ as a function of time $t$ for $g_{00}=g_{11}=g=40, g_{01}=g_{10}=0$ (red squares), $g_{00}=g_{11}=g=39.5$, $g_{01}=g_{10}=0.5$ (green circles), $g_{00}=g_{11}=g=28, g_{01}=g_{10}=12$ (blue diamonds), and $g_{00}=g_{11}=g_{01}=g_{10}=g=20$ (orange triangles). Other parameters are as in main part of Fig. 1.

$t_{s}$ can be rather large (predicted to be $t_{s} \sim g^{4} / k^{2}$ in [8]), this implies that the qubit can remain pure for a very long time.

In the regime where the qubit purity is high, the probability oscillates between the two internal states of the BEC. This leads to oscillations of the polarization of the qubit $\xi_{z}=\operatorname{Tr}\left(\rho \sigma_{z}\right)$, which are clearly seen in Fig. 3. These oscillations remain stable with respect to small variations in coupling matrix elements $g_{i j}$ (e.g., $g_{10}=g_{01}=19<g_{00}=g_{11}=g$ $=21$ in Fig. 3). However for a significant asymmetry $g_{10}$ $=g_{01}=10, g_{00}=g_{11}=g=30$ the nonlinear shift strongly modifies the unperturbed Rabi frequency and the amplitude of oscillations of $\xi_{z}(t)$ becomes strongly suppressed even if the purity remains close to unity. In this regime the distance between the centers of the two solitons with the qubit in up and down states remains small compared to their size (see Fig. 4). Only for small values of $g_{i j}=g$ the purity starts to drop rapidly and the oscillations of $\xi_{z}(t)$ decay quickly due to dynamical decoherence induced by quantum chaos. When the coupling matrix has small off-diagonal elements [e.g., $g_{01}=g_{10} \leq 0.5$ (see Fig. 4)], the solitons for the two components move independently from each other and the purity drops to zero as soon as the solitons are separated.

To better analyze the properties of these qubit oscillations, we determined the variation in spectral density $S(\nu)$ of $\xi_{z}(t)$ as a function of $g$. This spectrum is shown in Fig. 5. For small values of $g$, the spectral density is very low since quantum chaos leads to a rapid decay of qubit oscillations and purity. In contrast, for larger values of $g \geq 10$ a prominent peak at the Rabi frequency $\nu_{R}=T \delta / \pi$ becomes well pronounced even if the soliton dynamics is strongly chaotic $(K=k T=6.8)$.

The interplay of quantum chaos and GPE nonlinearity and their effects on the purity of the qubit are displayed in Fig. 6. For small $g<10$, the purity is destroyed with the increase in the chaos strength represented by the parameter $K$. On the contrary, for $g \geq 10$ the purity remains close to 1 even for large values of $K$ when the soliton dynamics is strongly chaotic.

This behavior can be qualitatively understood by the following arguments. From Eqs. (1) and (2) the coupling energy

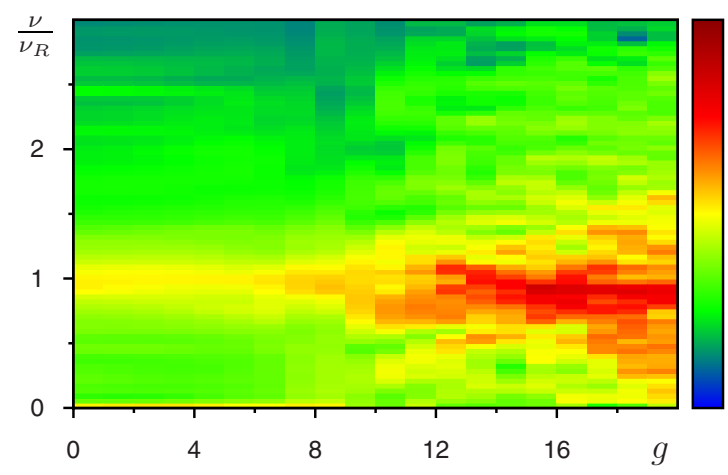

FIG. 5. (Color online) Density plot of the spectral density $S(\nu)$ of $\xi_{z}(t)$ expressed in arbitrary units and shown as a function of the frequency $\nu$ and the nonlinear parameter $g$ for $k=3.4, \varepsilon=0.5$, $T=2, \delta=0.2$, and the rescaled Rabi frequency $\nu_{R}=T \delta / \pi$. Initial state is the same as in Fig. 1. Color is proportional to $S(\nu)$ which varies from zero marked by blue/black to maximal value marked by $\mathrm{red} /$ gray.

of the single-component soliton at $g_{0}=g$ is $E_{s}=-g^{2} / 8$ [17]. For $g_{11} \approx g_{10} \approx g_{01} \approx g_{00} \approx g \gg 1$ the energy of attraction between the two components is of the same order of magnitude, thus being significantly larger than the difference $E_{k}$ between the kinetic energies induced by the kick term in Eq. (1). Indeed, the latter can be estimated as $E_{k} \sim p \Delta p \sim 2 k \varepsilon \sqrt{t}$; for $k \approx 3, t=20, \varepsilon=0,5$ this gives $E_{k} \approx 13$ and the condition $E_{s} \approx E_{k}$ gives the critical value of $g_{c} \approx 10$. This estimate for $g_{c}$ is compatible with the numerical results displayed in Fig. 6 even if this approach is rather simplified. In addition, we should also note that according to the well-known result [15] initial state (2) has a coupled solitonic state for $g>0.18 g_{0}$. For the conditions of Fig. 6 this gives a critical $g \approx 3.6$ that is significantly smaller than the numerical value of $g_{c} \approx 10$. We attribute this difference to the internal dynamics of qubit which is not taken into account by the estimate above. In the case $g_{11}=g_{00}=g \gg g_{01}=g_{10}$ the coupling energy between solitons can be obtained by degenerate perturbation theory which gives $E_{s} \sim-g_{01} g$. In the case where $E_{k}>E_{s}$, the solitons start to separate exponentially fast due to classical chaotic dynamics. Such kind of behavior can be potentially used to develop detectors with exponential sensitivity to the qubit state.

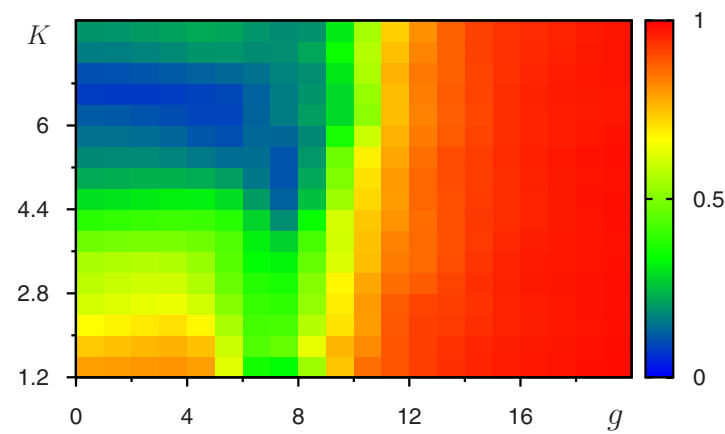

FIG. 6. (Color online) Density plot of the qubit purity $\xi$ after $t=20$ kicks as a function of the chaos parameter $K=k T$ and the nonlinear parameter $g$ for $T=2, \delta=0.2$, and $\varepsilon=0.5$. Initial state is the same as in Fig. 1. 
To realize experimentally model (1) described above, it is possible to use a typical ${ }^{87} \mathrm{Rb} \mathrm{BEC}$ and couple the hyperfine ground states $|0\rangle \equiv\left|F=1, m_{F}=-1\right\rangle$ and $|1\rangle \equiv\left|F=2, m_{F}=1\right\rangle$ via a two-photon (microwave +rf) transition, as is done in several experiments $[10,18]$. In this system the strength of the kick already depends on the orientation of the qubit because the hyperfine splitting of about $6.8 \mathrm{GHz}$ in ${ }^{87} \mathrm{Rb}$ induces a difference in detunings between the frequency of the lasers creating the pulsed optical lattice and the $D_{2}$ line, which gives rise to different dipole potential amplitudes for the $|0\rangle$ and $|1\rangle$ states. Moreover, the long coherence lifetime achieved in this system (2.8 s [18]) exceeds the typical duration of kicked BEC experiments [1] and would allow to observe the fast induced decoherence predicted here.

In conclusion, our results show that the coupling of a qubit with a chaotic BEC dynamics leads to rather nontrivial behavior. On one hand, the qubit can control the dynamics of the BEC, moving it from chaotic to integrable regimes and vice versa. The BEC position is exponentially sensitive to the state of the qubit. In spite of chaotic motion of BEC, the purity of the qubit is well preserved in this situation. Such an exponential sensitivity of a global BEC position to an internal quantum state appears due to the absence of second quantization for BEC motion in GPE. In presence of such quantization the whole system is described by a linear Schrödinger equation and the chaotic dynamics of BEC can continue only on a relatively short Ehrenfest time scale as it is discussed in [9]. On the other hand, in the regime of weak BEC nonlinearity, quantum chaos leads to rapid dynamical decoherence of the qubit. The realization of such coupling between a qubit and a BEC with chaotic dynamics is within reach of current experimental techniques.

We thank D. Guéry-Odelin and T. Lahaye for useful discussions. We thank CalMiP for access to their supercomputers and the French ANR (project INFOSYSQQ) and the EC project EuroSQIP for support. J.M. thanks the Belgian F.R.S.-FNRS for financial support.
[1] C. Ryu, M. F. Andersen, A. Vaziri, M. B. d'Arcy, J. M. Grossman, K. Helmerson, and W. D. Phillips, Phys. Rev. Lett. 96, 160403 (2006).

[2] G. Behinaein, V. Ramareddy, P. Ahmadi, and G. S. Summy, Phys. Rev. Lett. 97, 244101 (2006).

[3] S. A. Wayper, W. Simpson, and M. D. Hoogerland, EPL 79, 60006 (2007).

[4] M. Sadgrove, M. Horikoshi, T. Sekimura, and K. Nakagawa, Phys. Rev. Lett. 99, 043002 (2007).

[5] B. V. Chirikov, Phys. Rep. 52, 263 (1979); B. V. Chirikov, F. M. Izrailev, and D. L. Shepelyansky, Sov. Sci. Rev., Sect. C, Math. Phys. Rev. 2, 209 (1981); Physica D 33, 77 (1988); B. Chirikov and D. Shepelyansky, Scholarpedia J. 3, 3550 (2008).

[6] F. L. Moore, J. C. Robinson, C. F. Bharucha, B. Sundaram, and M. G. Raizen, Phys. Rev. Lett. 75, 4598 (1995).

[7] F. Dalfovo, S. Giorgini, L. P. Pitaevskii, and S. Stringari, Rev. Mod. Phys. 71, 463 (1999).

[8] F. Benvenuto, G. Casati, A. S. Pikovsky, and D. L. Shepelyansky, Phys. Rev. A 44, R3423 (1991).

[9] J. Martin, B. Georgeot, and D. L. Shepelyansky, Phys. Rev.
Lett. 101, 074102 (2008).

[10] J. Williams, R. Walser, J. Cooper, E. A. Cornell, and M. Holland, Phys. Rev. A 61, 033612 (2000).

[11] K. C. Wright, L. S. Leslie, A. Hansen, and N. P. Bigelow, Phys. Rev. Lett. 102, 030405 (2009).

[12] D. Sokolovski and S. A. Gurvitz, Phys. Rev. A 79, 032106 (2009).

[13] H. Ammann and N. Christensen, Phys. Rev. E 57, 354 (1998).

[14] J. W. Lee, D. V. Averin, G. Benenti, and D. L. Shepelyansky, Phys. Rev. A 72, 012310 (2005).

[15] V. E. Zakharov and A. B. Shabat, Zh. Eksp. Teor. Fiz. 61, 118 (1971) [Sov. Phys. JETP 34, 62 (1972)]; V. E. Zakharov, S. V. Manakov, S. P. Novikov, and L. P. Pitaevskii, Theory of Solitons (Nauka, Moscow, 1980) (in Russian).

[16] J. Laskar and P. Robutel, Celest. Mech. Dyn. Astron. 80, 39 (2001).

[17] L. D. Landau and E. M. Lifshitz, Quantum Mechanics (Nauka, Moscow, 1989).

[18] P. Treutlein, P. Hommelhoff, T. Steinmetz, T. W. Hänsch, and J. Reichel, Phys. Rev. Lett. 92, 203005 (2004). 